\title{
An overview on potential energy surfaces of rare-gas dihalogen van der Waals clusters
}

\author{
Gerardo Delgado-Barrio, Rita Prosmiti, Álvaro Valdés \\ and Pablo Villarreal \\ Instituto de Matemáticas y Física Fundamental, CSIC, Serrano 123, 28006 Madrid, Spain \\ E-mail: rita@imaff.cfmac.csic.es
}

Received 13 May 2005

Accepted for publication 24 September 2005

Published 21 December 2005

Online at stacks.iop.org/PhysScr/73/C57

\begin{abstract}
In this review we report on interaction potential surface calculations of $\operatorname{Rg}-X Y(\operatorname{Rg}=$ rare gas and $X, Y=$ halogens) van der Waals (vdW) complexes. Experimental data available on the structure and dynamics of such systems mainly originate from the $\mathrm{B} \leftarrow X$ excitation spectroscopy and, therefore, potential surfaces for both electronic states involved are required for the theoretical treatments. Hence, ab initio technology is used at the coupled-cluster $(\mathrm{CCSD}(\mathrm{T}))$ level of theory for constructing these surfaces. Relativistic effects are included with the use of large-core pseudo-potentials for the halogen atoms, while efficient augmented correlation-consistent polarized basis sets are employed for the $\mathrm{Rg}$ ones, to ensure saturation in interaction energies in the highest level of electron correlation treatment. For all ground state Rg-dihalogen systems studied, the potential surface shows minima for both linear and T-shaped orientations. In contrast, the potential surfaces of the electronically B excited state complexes present T-shaped minimum. Variational calculations for both electronic potentials are performed to calculate the bound states of the ground and B excited vdW complexes, and binding energies, vibrationally averaged structures and spectral shifts are determined. Here, an application of the present methodology on the ground $X$ and $\mathrm{B}$ excited $\mathrm{HeI}_{2}$ conformers is reported and the obtained results are discussed in terms of available experimental data.
\end{abstract}

PACS numbers: 31.15.Ar, 31.50.Bc, 31.50.Df, 33.15.Fm, 33.20.Vq, 36.40.Mr

\section{Introduction}

For more than three decades, van der Waals (vdW) complexes have become prototypes for studying energy transfer mechanisms and weak intermolecular forces $[1,2]$. During these years the understanding of $\mathrm{vdW}$ forces has expanded dramatically. With the development of experimental techniques, such as supersonic nozzle expansion, and by performing more accurate $a b$ initio electronic structure calculations, it became possible to study the structure and dynamics of vdW in more detail.

In this paper we present potential surface for ground and electronically excited $\mathrm{HeI}_{2}$ complexes, as obtained by coupled cluster $(\operatorname{CCSD}(\mathrm{T}))$ calculations [3, 4]. This forms part of an extended investigation of the interactions between rare gas atoms and homonuclear or heteronuclear dihalogens. For ground state rare gas-halogens, there is a general acceptance of the existence of a double-minimum topology for linear and T-shaped isomers, based on recent experimental studies [5-10] and a series of ab initio calculations [3, 12-24]. In the case of $\mathrm{B}$ excited complexes, experimental measurements $[8,25]$ and theoretical studies $[4,25,26]$ have established a T-shaped structure. The experimental data available on the structure and dynamics of these systems originate mainly from the $\mathrm{B} \leftarrow X$ excitation spectroscopy [8, 25], and the potential energy surfaces (PESs) for both electronic states are required for theoretical simulations. There is a plethora of $a b$ initio studies of such complexes focused on the topology of their ground PESs, while due to the difficulties in applying high level $a b$ initio methods for an open-shell state fewer $a b$ initio results are available for the B excited electronic state of the rare gas-dihalogens. Only recently $a b$ initio calculations have been reported in the literature for the $\mathrm{B}$ states of the $\mathrm{HeCl}_{2}$ [25], $\mathrm{HeBr}_{2}$ [26] and $\mathrm{HeI}_{2}$ [4], while semiempirical 
potentials are still used in theoretical studies for the B state dynamics of similar systems. It still remains a challenge to obtain accurate potential energy functions for such molecules and to interpret the dynamics involved [25].

$\mathrm{HeI}_{2}$ was the first rare gas-dihalogen vdW molecule studied by Levy and co-workers [27, 28]. Their analysis indicates that the molecule on both ground and B excited state has a nonlinear structure and vibrationally average configurations have been determined. The other data available from these studies are blue-shift values for low and high $v$ levels [28, 29] and the $X$ and B binding energies [28, 30]. Recent experimental studies by Loomis and co-workers in the $\mathrm{B} \leftarrow X$ spectrum have shown that spectral features are associated with transitions of multiple conformers of several $\mathrm{He}-X Y$ complexes [8], and binding energies and structures of such isomers reported for $\mathrm{HeI}_{2}$ [31].

The first attempt to calculate a purely ab initio surface for $\mathrm{HeI}_{2}$ complex was undertaken by Schwenke and Truhlar [32] for its ground electronic state. The authors have calculated, using Möller-Plesset perturbation theories up to third order, a significantly shallow well, of $6 \mathrm{~cm}^{-1}$, for T-shaped geometries. Recently, $\operatorname{CCSD}(\mathrm{T})$ ab initio calculations have been performed for the ground and B excited electronic states of $\mathrm{HeI}_{2}$ complex [3, 4], and binding energies and structures of linear and T-shaped isomers have been determined.

To our knowledge, no more ab initio studies on the PESs of the heavy $\mathrm{HeI}_{2}$ complex are reported in the literature. Thus, we summarize here, the methodology for constructing ab initio potential surfaces of $\mathrm{Rg}$-dihalogen complexes and an application to $\mathrm{HeI}_{2}$ is presented. We should note that a direct comparison with experiment requires theoretical data of the same quality for both electronic surfaces involved. Hence, by combining both $X$ and B PES results will allow us to make the first comparison between completely ab initio calculations and experimental data.

\section{Methodology and results}

\subsection{Ab initio computation}

We use Jacobi coordinates $(r, R, \theta)$ to describe the potential surfaces of $\operatorname{Rg}-X Y$ complexes, where $R$ is the intermolecular distance of $\mathrm{Rg}$ atom from the centre of mass of $X Y, r$ is the bond length of $X Y$ and $\theta$ is the angle between the $\boldsymbol{R}$ and $\boldsymbol{r}$ vectors.

The $a b$ initio calculations for the $X$ state are performed using the Gaussian 98 package [33], using the spin-restricted single and double excitations coupled cluster method with perturbative triples $(\operatorname{RCCSD}(\mathrm{T}))$, while for the first excited triplet state B the MOLPRO package ${ }^{1}$ is used at spinunrestricted $\operatorname{UCCSD}(\mathrm{T})$ level of theory. In the case of homonuclear $X Y$ dihalogen, the degeneracy of the $\mathrm{B}\left({ }^{3} \Pi\right)$ excited state is removed by the $\mathrm{Rg}$ atom approaching giving rise to two states ${ }^{3} A^{\prime}$ and ${ }^{3} A^{\prime \prime}$. In the ${ }^{3} A^{\prime}$ Rg faces a single occupied $\pi^{*}$ orbital while in ${ }^{3} A^{\prime \prime}$ a doubly occupied one. The states are expected to be very close to each other since the perturbation exerted by $\mathrm{Rg}$ is small. In addition, when $\mathrm{Rg}$ rotates from the $\mathrm{T}$-shaped geometry to the collinear one,

1 MOLPRO is a package of $a b$ initio programs written by H-J Werner and P J Knowles, with contributions from R D Amos et al. these two states converge and are degenerate at the linear configurations. We should note that the individual ${ }^{3} A^{\prime}$ and ${ }^{3} A^{\prime \prime}$ adiabatic states do not exist separately, since the spin-orbit couples them. In the present calculations of the B state, spinorbit coupling terms are not included, due to the very large computational effort required.

For all the calculations of the $\operatorname{Rg}-X Y$ potentials, we use effective core potentials basis set for the heavy $X$ and $Y$ halogen atoms, which is a convenient method to incorporate relativistic effects [34] in standard quantum mechanical calculations. We employ a large-core energyconsistent pseudo-potential [35] in conjunction with an augmented correlation consistent valence basis set [34] for halogen atoms. For $\mathrm{Rg}$ atoms, augmented correlation consistent basis sets are taken from the EMSL library [36]. In the $X$ state calculations, an additional set $(3 \mathrm{~s} 3 \mathrm{p} 2 \mathrm{~d} 2 \mathrm{f} 1 \mathrm{~g})$ of bond functions is employed [37].

The intermolecular energy between $\mathrm{Rg}$ and $X Y$ is calculated using the supermolecular approach. The interaction energy, $\Delta E$, is given as the difference between the total energy of the complex, $E_{\operatorname{Rg} X Y}$, and the sum of the energies of the monomers, $E_{\mathrm{Rg}}+E_{X Y}$. The standard counterpoise method [38] is used for the correction of basis set superposition error (BSSE).

Results are presented here for the $\mathrm{HeI}_{2}$ complex, where the intermolecular energies are calculated for several $R$ distances ranging from $R=3$ to $9 \AA$, while the angle $\theta$ is varied between $0^{\circ}$ and $90^{\circ}$ on a seven equally spaced (by $\pi / 12$ radians) grid, considering four different $\mathrm{I}_{2}$ bond lengths with $r=2.42249,2.666,2.90951$ and $3.11092 \AA$ for the ground state and $r=2.65,2.85,3.024$ and $3.20 \AA$ for the $\mathrm{B}$ excited state. The $r$ values are chosen around the equilibrium distances in a range that is enough to describe some of the first excited vibrational levels of $\mathrm{I}_{2}(X)$ and $\mathrm{I}_{2}(\mathrm{~B})$.

The results of the interaction energies for the $X$ state for different I-I bond lengths are qualitatively similar. For all the $r$ values, the linear configuration is found to be lower in energy than the T-shaped one. When the I-I bond is lengthened their energy difference increases. We should note that the interaction energies of the T-shaped structures are found to be more sensitive to small changes of the I-I bond length than the ones for the linear configurations. Also, it is useful to examine the $A^{\prime} / A^{\prime \prime}$ interaction energies for the different I-I bond lengths. For small values of $r=2.65$ and $2.85 \AA$, there is only one minimum for the T-shaped structure for both $A^{\prime}$ and $A^{\prime \prime}$ surfaces. For elongated $r$ values ( $r=3.024,3.20$ and $3.45 \AA$ ) the $A^{\prime}$ surface presents only a T-shaped minimum, while the $A^{\prime \prime}$ presents two minima for T-shaped and linear structures. In this sense, the results of the $A^{\prime \prime}$ interaction energies are very similar to the $X$ electronic state of the complex.

\subsection{Representation of PESs of $\mathrm{HeI}_{2}$}

In order to represent the $X$ and $\mathrm{B}$ PESs for a $\operatorname{Rg}-X Y$ complex, an analytical functional form is used to fit the CCSD(T) ab initio points. As noted previously, for an accurate representation of the $\mathrm{B}$ state potential, one should take into account the spin-orbit coupling for the $A^{\prime}$ and $A^{\prime \prime}$ adiabats. However, in the atom-diatom case, the matrix element of 


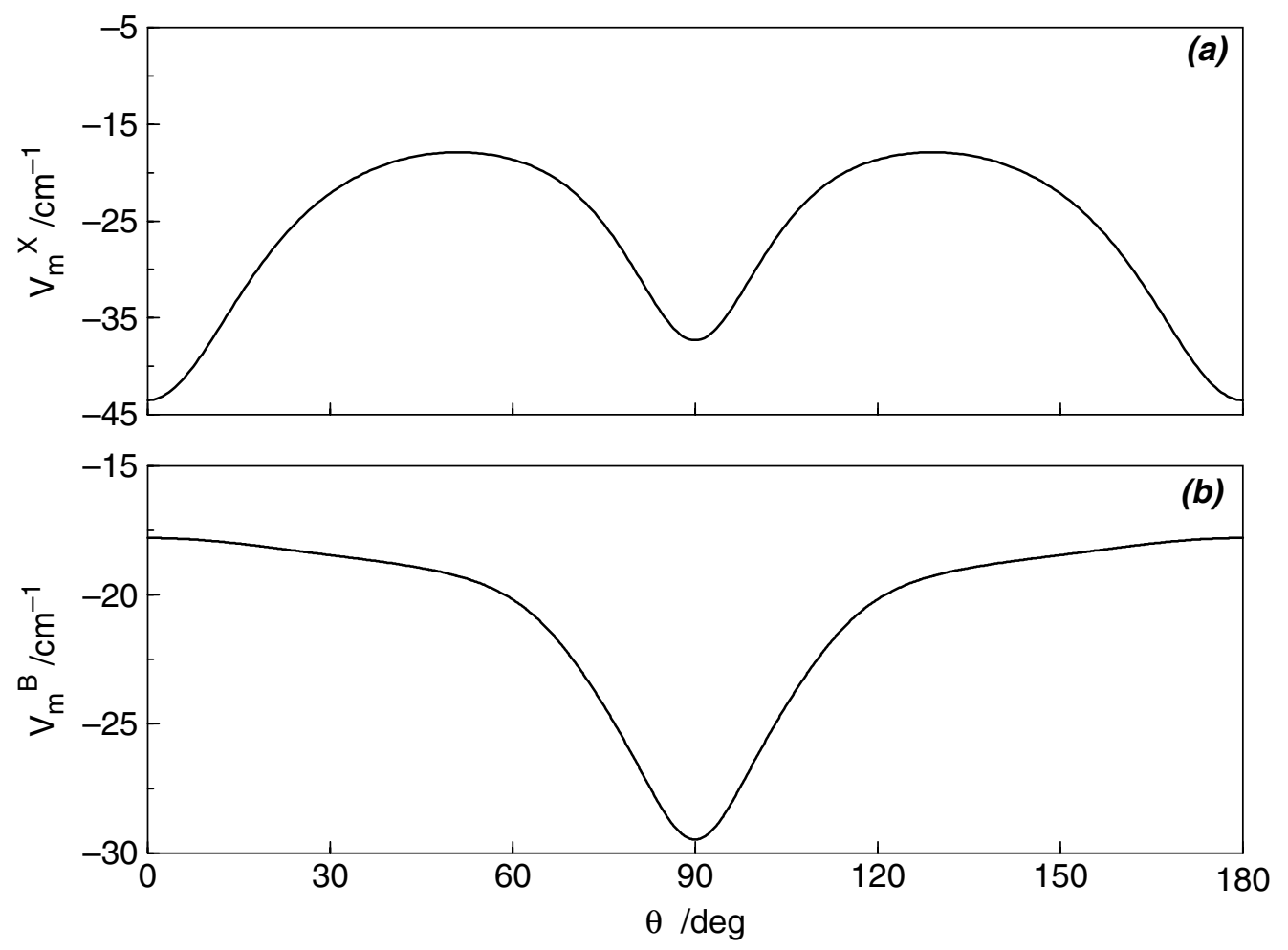

Figure 1. Minimum energy paths (a) $V_{m}^{X}$ and (b) $V_{m}^{B}$ in $\mathrm{cm}^{-1}$ as a function of $\theta$.

the interaction potential can be represented as the sum or difference of the $A^{\prime}$ and $A^{\prime \prime}$ potentials [39, 40]. Here, we represent the $\mathrm{B}$ state potential as the average of the $A^{\prime}$ and $A^{\prime \prime}$ states, $V_{B}=A^{\prime}+A^{\prime \prime} / 2$.

We choose an expansion in Legendre polynomials, $P_{\lambda}(\cos \theta)$, to describe both the two-dimensional (2D) $\operatorname{Rg} \cdots X Y(X)$ and $\operatorname{Rg} \cdots X Y(\mathrm{~B})$ interaction potentials,

$$
V_{X, B}\left(R, \theta ; r_{k}\right)=\sum_{\lambda} V_{k \lambda}^{X, B}(R) P_{\lambda}(\cos \theta), \quad k=1-4,
$$

with $\lambda=0,2,4,6,8,10$ and 12 , due to the symmetry of the system with respect to $\theta=90^{\circ}$. The $V_{k \lambda}^{X, B}(R)$ coefficients are obtained by a collocation method applying the following procedure. For each electronic state for each of the seven values of angle $\theta$, we fitted the $\mathrm{R} / \mathrm{UCCSD}(\mathrm{T})$ data to a Morse-vdW function,

$$
\begin{gathered}
V_{X, B}\left(R ; \theta_{i} ; r_{k}\right)=\alpha_{0, i k}^{X, B}\left\{\exp \left[-2 \alpha_{1, i k}^{X, B}\left(R-\alpha_{2, i k}^{X, B}\right)\right]\right. \\
\left.-2 \exp \left[-\alpha_{1, i k}^{X, B}\left(R-\alpha_{2, i k}^{X, B}\right)\right]\right\}-\frac{\alpha_{3, i k}^{X, B}}{R^{6}}-\frac{\alpha_{4, i k}^{X, B}}{R^{8}}
\end{gathered}
$$

with parameters $\alpha_{0, i k}^{X, B}, \alpha_{1, i k}^{X, B}, \alpha_{2, i k}^{X, B}, \alpha_{3, i k}^{X, B}$ and $\alpha_{4, i k}^{X, B}$, where $i=1-7$ and $k=1-4$, obtained from nonlinear least square procedure.

The ground $\mathrm{HeI}_{2}$ PES exhibits two minima. The global minimum with an energy of $-43.52 \mathrm{~cm}^{-1}$ at $R_{X}=4.89 \AA$ corresponds to a linear $\left(\theta=0^{\circ}\right)$ configuration. The second minimum with an energy of $-37.32 \mathrm{~cm}^{-1}$ at $R_{X}=3.84 \AA$ corresponds to a T-shaped $\left(\theta=90^{\circ}\right)$ configuration of the complex. The isomerization barrier between the two wells is found at energy of $-17.88 \mathrm{~cm}^{-1}\left(25.64 \mathrm{~cm}^{-1}\right.$ above the global linear minimum), with $R_{X}=4.93 \AA$ and $\theta \sim 51^{\circ}$. For the $\mathrm{B}$ excited state the global minimum corresponds to a T-shaped configuration with energy $-29.48 \mathrm{~cm}^{-1}$ at $\mathrm{R}_{B}=$ $3.96 \AA$. These potential minima for both electronic states are displayed in figure 1 , where the minimum energy paths, $V_{m}^{X}$ and $V_{m}^{B}$, are plotted as a function of the angle $\theta$.

\subsection{Bound state calculations}

It should be stressed that for an accurate dynamic treatment of the B state complex an effective Hamiltonian, which includes the spin-orbit coupling term, should be employed following the formalism given in $[39,40]$. In the present case, the ab initio calculations reported provide the adiabatic $A^{\prime}$ and $A^{\prime \prime}$ potentials for the $\mathrm{B}$ state, thus a spin-free Hamiltonian has been used for both $X$ and B states. The rovibrational Hamiltonian in the Jacobi coordinate system has the form

$$
\begin{aligned}
\hat{H}^{X, B}= & -\frac{\hbar^{2}}{2 \mu_{1}} \frac{\partial^{2}}{\partial R^{2}}+\frac{\hat{j}^{2}}{2 \mu_{2} r^{2}}+\frac{\hat{l}^{2}}{2 \mu_{1} R^{2}} \\
& +V_{X, B}(R, \theta, r)+\hat{H}_{I_{2}}^{X, B},
\end{aligned}
$$

where $\hat{H}_{I_{2}}^{X, B}=-\hbar^{2} / 2 \mu_{2} \partial^{2} / \partial r^{2}+V_{I_{2}}^{X, B}(r)$ is the vibrational Hamiltonian for a free $X Y$ molecule. $1 / \mu_{1}=$ $1 / m_{\mathrm{Rg}}+1 / m_{\mathrm{X}}+m_{\mathrm{Y}}$ and $1 / \mu_{2}=1 / m_{\mathrm{X}}+1 / m_{\mathrm{Y}}$ are the reduced masses. In $\mathrm{HeI}_{2}$ case $m_{\mathrm{He}}=4.00260$ and $m_{\mathrm{I}}=126.904473 \mathrm{amu}$ are the atomic masses of ${ }^{4} \mathrm{He}$ and ${ }^{127} \mathrm{I}$ isotopes, $\hat{l}$ and $\hat{j}$ are the angular momentum operators associated with the vectors $\boldsymbol{R}$ and $\boldsymbol{r}$, respectively, leading to a total angular momentum $\hat{J}=\hat{l}+\hat{j}$. Here all calculations are performed for $J=0$. Starting from the $V_{X, B}\left(R, \theta ; r_{k}\right)$ potential of (equation (1)), 1D cubic-spline interpolation is used to compute the value of $V_{X, B}(R, \theta, r)$ at 21 Gauss-Legendre 
Table 1. Experimental and theoretical binding energies $\left(D_{e}\right.$ and $D_{0}$ in $\left.\mathrm{cm}^{-1}\right)$, equilibrium distances $\left(R_{e}\right.$ and $R_{0}$ in $\AA$ ) and frequencies, for the linear and T-shaped isomers of the ground and excited $\mathrm{HeI}_{2}$ complexes.

\begin{tabular}{|c|c|c|c|c|c|c|c|c|}
\hline & \multicolumn{4}{|l|}{ Linear } & \multicolumn{4}{|l|}{ T-shaped } \\
\hline & $D_{e}$ & $D_{0}$ & $R_{e} / R_{0}$ & $\omega_{e} / \omega_{e} x_{e}$ & $D_{e}$ & $D_{0}$ & $R_{e} / R_{0}$ & $\omega_{e} / \omega_{e} x_{e}$ \\
\hline \multicolumn{9}{|l|}{$\overline{\mathrm{HeI}_{2}(\mathrm{X})}$} \\
\hline This work & 43.52 & 15.38 & $4.89 / 5.34$ & $8.12 /-$ & 37.32 & 14.68 & $3.84 / 4.40$ & $6.71 /-$ \\
\hline 1D CCSD(T) Morse & 44.24 & 23.87 & $4.88 /-$ & $46.97 / 12.47$ & 37.82 & 21.40 & $3.84 /-$ & $37.48 / 9.29$ \\
\hline Ab initio MP2/MP3 [32] & - & - & - & - & $6.29 / 6.94$ & - & $4.45 /-$ & - \\
\hline Semiempirical value [32] & - & - & - & - & 22.02 & - & $4.05 /-$ & - \\
\hline Semiempirical value $^{\mathrm{a}}[42]$ & 52.1 & 33.1 & $4.24 /-$ & $42.22 / 8.55$ & - & - & - & - \\
\hline Experimental value $[27,28]$ & - & - & - & - & $22.15 \pm 0.55$ & $18.8 \pm 0.6$ & $3.94 / 4.47$ & $6.95-7.09 / 0.53-0.58$ \\
\hline Experimental value [30] & - & - & - & - & - & $17.6 \pm 1.0$ & - & - \\
\hline Experimental value [31] & - & $17.3 \pm 0.5$ & - & - & - & $17.8 \pm 1.0$ & - & - \\
\hline \multicolumn{9}{|l|}{$\mathrm{HeI}_{2}(\mathrm{~B})$} \\
\hline This work & & & & & 29.48 & 12.33 & $3.96 / 4.58$ & $3.97 /-$ \\
\hline 1D CCSD(T) Morse & & & & & 29.72 & 16.26 & $3.97 / 4.32$ & $30.96 / 8.06$ \\
\hline Semiempirical value $[1,45]$ & & & & & 26.00 & 14.57 & $4.0 / 4.38$ & $26.15 / 6.58$ \\
\hline Experimental value $[27,28]$ & & & & & $17.55 \pm 0.55$ & $14.2 \pm 0.6$ & $4.79 \pm 0.22$ & $7.02-7.18 / 0.68-0.76$ \\
\hline Experimental value $^{\mathrm{b}}[31]$ & - & - & - & - & - & $14.1 \pm 1.0$ & - & - \\
\hline
\end{tabular}

${ }^{a}$ We assume that these potential parameters are for the $\mathrm{HeI}_{2}$ linear structure, since they have been used to study atom-diatomic molecule collinear collisions.

${ }^{\mathrm{b}}$ This value is for the binding energy of $\mathrm{HeI}_{2}(\mathrm{~B}, v=20)$.

points in the interval of $r_{\min }^{X, B}<r^{X, B}<r_{\max }^{X, B} . V_{I_{2}}^{X, B}(r)$ are the $1 D \mathrm{I}_{2}(X)$ or $\mathrm{I}_{2}(\mathrm{~B})$ state potential functions and a cubic-spline interpolation to $\operatorname{CCSD}(\mathrm{T})$ ab initio data is used to reproduce the $V_{I_{2}}^{X, B}$ potential at any $r$ point. The eigenvalues and eigenfunctions of diatomic $\hat{H}_{I_{2}}$ Hamiltonian are denoted as $E_{I_{2}}(v)$ and $\chi_{v}(r)$, respectively, and are evaluated by solving the 1D Schrödinger equation using a combined Truhlar-Numerov algorithm [41]. The vdW levels and corresponding wave functions are calculated variationally by diagonalizing the vibrationally averaged Hamiltonian

$$
\begin{aligned}
H_{v}^{X, B}= & \left\langle\chi_{v}\left|H^{X, B}\right| \chi_{v}\right\rangle \\
= & -\frac{\hbar^{2}}{2 \mu_{1}} \frac{\partial^{2}}{\partial R^{2}}+\frac{\hat{l}^{2}}{2 \mu_{1} R^{2}}+V_{v, v}^{X, B}(R, \theta) \\
& +E_{I_{2}}^{X, B}(v)+\frac{B_{v}^{X, B} \hat{j}^{2}}{\hbar^{2}},
\end{aligned}
$$

where $V_{v, v}^{X, B}(R, \theta)=\left\langle\chi_{v}\left|V^{X, B}(R, \theta, r)\right| \chi_{v}\right\rangle$ is the intermolecular vdW potential of $\mathrm{HeI}_{2}(X, \mathrm{~B})$ averaged over the $\mathrm{I}_{2}(X, \mathrm{~B})$ $v=0$ vibrational eigenfunction and $B_{v}^{X, B}$ is the $\mathrm{I}_{2}(X, \mathrm{~B})$ average rotational constant. The Hamiltonian is represented on a finite $3 \mathrm{D}$ basis set. The $V_{v, v}^{X, B}$ potential matrix elements are calculated using Gaussian quadrature in the $r$ coordinate, while for the angular coordinate we used orthonormalized Legendre polynomials $\left\{P_{j}(\cos \theta)\right\}$ as basis functions, with up to 40 values (even and odd) of the diatomic rotation $j$. For the radial $R$ coordinate, a discrete variable representation (DVR) basis set is used based on the particle in a box eigenfunctions [43]. A basis set of 75 DVR functions over the range from $R=1.75$ to $15 \AA$ are used. In this way, a convergence of $0.00005 \mathrm{~cm}^{-1}$ is achieved in bound state calculations.

In table 1 we compare the results obtained with the present $\operatorname{CCSD}(\mathrm{T})$ surface with previous theoretical and experimental data available for both $X$ and B electronic states. In addition, some semiempirical data are also available on the potential parameters for an interaction potential for the collinear collision of $\mathrm{He}$ atom and diatomic $\mathrm{I}_{2}$ oscillator, thus we compare these values with the ones corresponding to the present linear potential well (see table 1). The angular and radial probability distributions of the associated wave functions for the $X$ and B states are shown in figures 2 and 3, respectively.

Direct experimental data are available only for the $D_{0}$ value of the B state, and the spectral blue-shift value with respect to the corresponding band of the uncomplexed iodine molecule [44]. The experimental $D_{0}^{B}$ value [28] is between 13.6 and $14.8 \mathrm{~cm}^{-1}$ and compares very well with the present estimate of $D_{0}^{B}=12.33 \mathrm{~cm}^{-1}$. Based on the above measurements, the experimental value for binding energy of the $\mathrm{X}$ state of $\mathrm{HeI}_{2}$ has been determined [28] to be in the range $18.2-19.4 \mathrm{~cm}^{-1}$. When Blazy et al [28] reported the above values for $D_{0}$ they did not determine accurate values for the blue shifts of the $\mathrm{HeI}_{2} \mathrm{~B} \leftarrow X$ excitation spectra for the high $v$ levels. These shifts have been measured later by Sharfin et al and a lower value (by $1.2 \mathrm{~cm}^{-1}$ ) than the one used by Blazy et al has been obtained for the $v=62$ level. Thus, a revised value of $17.6 \pm 1.0 \mathrm{~cm}^{-1}$ has been proposed for the $D_{0}$ of the ground $\mathrm{HeI}_{2}$ [30]. We should note that our prediction of $15.38 \mathrm{~cm}^{-1}$ for the $D_{0}$ binding energy is very close to the lower bound of the later experimental value (see table 5). The present value, however, corresponds to a linear configuration, although we should stress that the support by the above experiment for a nonlinear structure is based on a sampling of alternatives (see reference [11] of [27]). Further, recent experimental values are available [31] for the binding energies of ground state isomers, which count $D_{0}^{L}=$ $17.3 \pm 0.5 \mathrm{~cm}^{-1}$ and $D_{0}^{T}=17.8 \pm 1.0 \mathrm{~cm}^{-1}$ for linear and T-shaped conformers, respectively. As can be seen (table 1), a reverse order of the two isomers is predicted by the $a b$ initio calculations. However, a very small energy difference between them has also been obtained experimentally, which is smaller than the error bars in the measured binding energies. Additionally, Loomis and co-workers based on their X state binding energy and the shift of the T-shaped feature from the band origin of the spectrum, have also determined a binding 

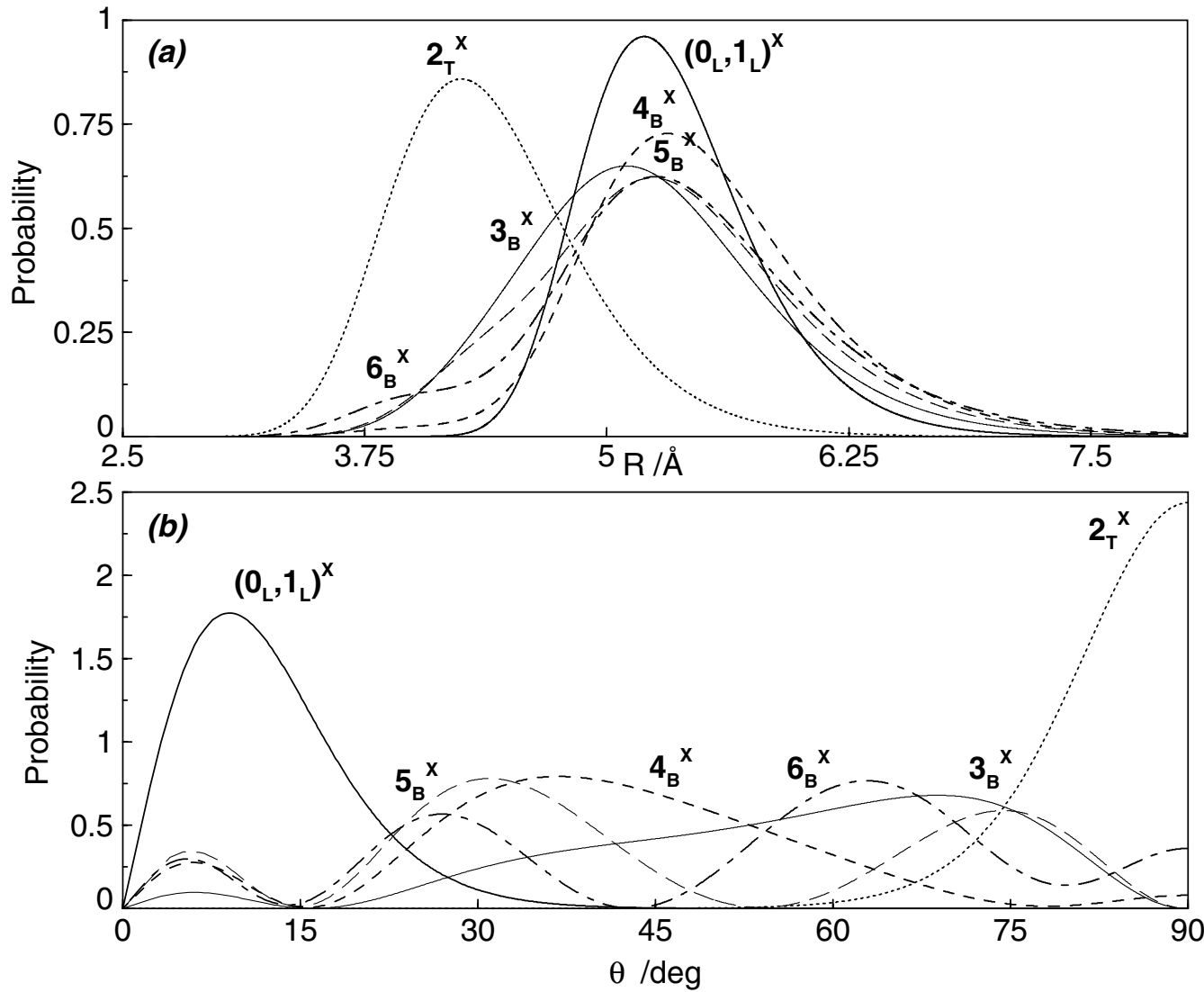

Figure 2. Radial and angular probability distributions (a, b) for the indicated $n_{C}^{X}$ vdW levels, calculated using the $V_{X}(R, \theta, r) \operatorname{PES}$. L $=$ linear, $\mathrm{T}=\mathrm{T}$-shaped and $\mathrm{B}=$ bending configurations. Angular distributions contain the Jacobian $\sin \theta$ volume element.

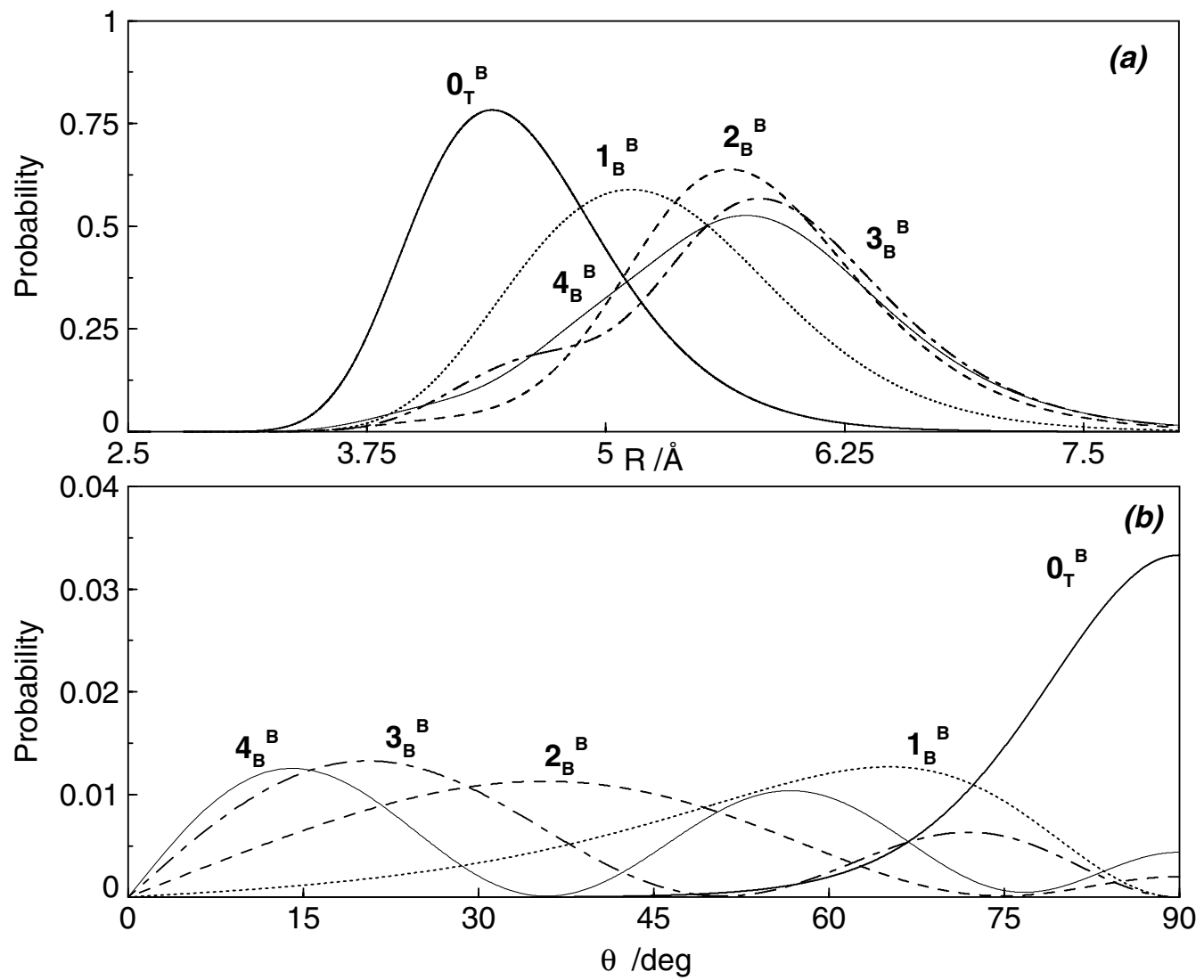

Figure 3. Radial and angular probability distributions (a,b) for the indicated $n_{C}^{B}$ vdW levels, calculated using the $V_{B}(R, \theta, r) \mathrm{PES}$. T $=$ T-shaped and $\mathrm{B}=$ bending configurations. Angular distributions contain the Jacobian $\sin \theta$ volume element. 
energy of $14.1 \pm 1.0 \mathrm{~cm}^{-1}$ for the T-shaped $\mathrm{HeI}_{2}(\mathrm{~B}, v=20)$. This value is in the same range as the one reported by Levy and co-workers [28] and very close to the present estimate of $D_{0}^{B}=12.33 \mathrm{~cm}^{-1}$ obtained for the T-shaped $\mathrm{HeI}_{2}(\mathrm{~B}, v=0)$ conformer.

In turn, based on the present $a b$ initio calculations, an estimate of the $\mathrm{B} \leftarrow X$ excitation frequency blue shift is obtained by combining data for the $X$ and B potential surfaces. A blue-shift value of $3.05 \mathrm{~cm}^{-1}$ is calculated as the difference of the $D_{0}^{X}-D_{0}^{B}$. Taking into account Franck-Condon factors, that strongly favour the transition between the $2_{T}^{X}$ and $0_{T}^{B} \mathrm{vdW}$ levels, a value of $2.35 \mathrm{~cm}^{-1}$ is approximated as the difference of their $J=0$ energies. We should note that the selection rules for dipole allowed transitions are $\Delta J=0, \pm 1(0 \leftrightarrow 0)$. The experimental measurement [28] for $v=3$ is $3.44 \mathrm{~cm}^{-1}$, that is very close to the present theoretical estimates. Moreover, it is likely to have larger errors than the above one arising from the spin-orbit coupling effects in the B state potential.

As we discussed above, our calculations indicate the coexistence of the two isomers for the ground state complex in a supersonic beam, which is consistent with recent experimental observations [31]. However, given the difficulties of the experimental studies in determining equilibrium structures of different isomers, as well as the limitations of the $a b$ initio technology, it is clear that experimental studies similar to those reported recently $[8,9]$ on the LIF spectra and action spectroscopy experiments [10], in combination with theoretical simulations are invaluable for interpreting intermolecular dynamics of such complexes (see also [46]).

\section{Conclusions}

In this review we summarized the methodology for constructing reliable $a b$ initio PESs for ground and excited triatomic Rg-dihalogen vdW complexes. The main difficulty of the theory of weakly bound systems arises from the very delicate relationship between the global interaction PES's and experimental observations. Comparison of theoretical results with experimental data is subjected to the errors coming from the poor knowledge of the PES, the dynamical approximations and the uncertainties of the experimental measurements. For $\mathrm{Rg}$-dihalogen complexes, the route to accurate interaction PES's is the harder, as it depends on the complexity of the system. We have shown that using ab initio technology at the CCSD(T) level of theory provides PESs for the Rg-dihalogen complexes, that are able to reproduce quantitatively or at least semiquantitatively the available experimental data.

We present results of applying the present methodology on $\mathrm{HeI}_{2}$ complex, where 3D interaction PESs are calculated for the $\mathrm{HeI}_{2}(X)$ and $\mathrm{HeI}_{2}(\mathrm{~B})$ complexes at the $\operatorname{CCSD}(\mathrm{T})$ level of theory. As in other studies on such complexes, different topologies are obtained from the PESs of the $X$ and B electronic states. The $X$ potential surface exhibit double minima for linear and T-shaped configurations, while first excited triple state, B, shows a single T-shaped well. Bound state calculations with $J=0$ are carried out for both $X$ and $\mathrm{B} \operatorname{CCSD}(\mathrm{T})$ surfaces, and binding energies, vibrationally averaged structures and spectral shifts are calculated. For the ground state, the linear $\mathrm{He}-\mathrm{I}-\mathrm{I}$ and the T-shaped isomers are found to lie very close to each other, with a difference of only $0.7 \mathrm{~cm}^{-1}$. The above values, as well as the values for the B state are in very good accordance with early experimental observations [27] available for the perpendicular structure. A good agreement is also obtained between the $\operatorname{CCSD}(\mathrm{T})$ results and the experimental estimates $[28,30,31]$ concerning the $D_{0}^{X, B}$ values, although the ground linear He-I-I isomer is predicted here to be more stable than the corresponding T-shaped one.

These findings demonstrate that $\operatorname{CCSD}(\mathrm{T})$ calculations provide an alternative way of constructing reliable potential surfaces for such complexes, which could motivate detailed theoretical studies on the dissociation dynamics of $\mathrm{HeI}_{2}$. The work presented here has not taken into account spin-orbit coupling. Such relativistic effects are expected to influence the dynamics, and therefore work in this direction still remains. Further, the above description of the atom-diatomic molecule interaction is of considerable importance in the study of the structure and bonding in larger systems $\operatorname{Rg}_{n}-X Y$ [47, 48], where a diatomic molecule interacts with a solvent system of rare gas atoms, e.g. the relaxation dynamics of impurities embedded in He nanodroplets [49]. Whether the properties of the weak bonding in such systems can be predicted by the sum of atom-diatom interactions deserve further investigation.

\section{Acknowledgments}

The authors thank Centro de Calculo (IMAFF), CTI (CSIC), CESGA and GSC (CIEMAT) for allocation of computer time. This work has been supported by DGICYT, Spain (grant no FIS2004-0246). RP acknowledges a contract from the Comunidad Autónoma de Madrid, Spain.

\section{References}

[1] Beswick J A, Delgado-Barrio G and Jortner J 1979 J. Chem. Phys. 703895

[2] Levy D H 1981 Adv. Chem. Phys. 47323

[3] Prosmiti R, Valdés A, Villarreal P and Delgado-Barrio G 2004 J. Phys. Chem. 1086065

[4] Valdés A, Prosmiti R, Villarreal P, Delgado-Barrio G and Werner, H-J 2005 J. Chem. Phys. submitted

[5] Miller A E S, Chuang C-C, Fu H C, Higgins K J and Klemperer W 1999 J. Chem. Phys. 1117844

[6] Burroughs A and Heaven M C 2001 J. Chem. Phys. 1147027

[7] Burroughs A, Kerenskaya G and Heaven M C 2001 J. Chem. Phys. 115784

[8] Boucher D S, Bradke M D, Darr J P and Loomis R A 2003 J. Phys. Chem. 1076901

[9] Strasfeld D B, Darr J P and Loomis R A 2004 Chem. Phys. Lett. 397116

[10] Darr J P, Glennon J J and Loomis R A 2005 J. Phys. Chem. 122131101

[11] Rohrbacher A, Williams J, Janda K C, Cybulski S M, Burcl R, Szcześniak M M, Chalasiński G and Halberstadt N 1997 J. Chem. Phys. 1062685

[12] Williams J, Rohrbacher A, Djahandideh D, Janda K C, Jamka A, Tao F-M and Halberstadt N 1997 Mol. Phys. 91573

[13] Naumkin F Y and McCourt F R W 1998 Chem. Phys. Lett. 29471

[14] Higgins K, Tao F-M and Klemperer W 1998 J. Chem. Phys. 1093048

[15] Cybulski S M and Holt J S 1999 J. Chem. Phys. 1107745

[16] Prosmiti R, Villarreal P and Delgado-Barrio G 2002 Chem. Phys. Lett. 359473 
[17] Prosmiti R, Cunha C, Villarreal P and Delgado-Barrio G 2002 J. Chem. Phys. 1177017

[18] Valdés A, Prosmiti R, Villarreal P and Delgado-Barrio G 2003 Chem. Phys. Lett. 357328

[19] Prosmiti R, Cunha C, Villarreal P and Delgado-Barrio G 2003 J. Chem. Phys. 1194216

[20] Prosmiti R, Villarreal P and Delgado-Barrio G 2003 Israel J. Chem. 43297

[21] Tao F-M and Klemperer W 1992 J. Chem. Phys. 97440

[22] Kunz Ch F, Burghardt I and He B A 1998 J. Chem. Phys. 109359

[23] Cybulski S M and Holt J S 1999 J. Chem. Phys. 1107745

[24] Naumikin F Y 2001 Chem. Phys. Chem. 2121

[25] Williams J, Rohrbacher A, Seong J, Marianayagam N, Janda K C, Burcl R, Szczesniak M M, Chalasinski G, Cybulski S M and Halberstadt N 1999 J. Chem. Phys. 111997

[26] de Lara-Castells M P, Buchachenko A A, Delgado-Barrio G and Villarreal P 2004 J. Chem. Phys. 1202182

[27] Smalley R F, Wharton L and Levy D H 1978 J. Chem. Phys. 68671

[28] Blazy J A, DeKoven B M, Russell T D and Levy D H 1980 J. Chem. Phys. 722439

[29] Sharfin W, Kroger P and Wallace S C 1982 Chem. Phys. Lett. 8581

[30] Jahn D G, Clement S G and Janda K C 1994 J. Chem. Phys. 101283

[31] Loomis R A 2005 private communication

[32] Schwenke D W and Truhlar D G 1983 Chem. Phys. Lett. 98217
[33] Frisch M J et al 1998 Gaussian 98, Revision A.7 (Pittsburgh, PA: Gaussian Inc.)

[34] Martin J M L and Sundermann A 2001 J. Chem. Phys. 1143408

[35] Bergner A, Dolg M, Kuechle W, Stoll H and Preuss H 1993 Mol. Phys. 801431

[36] Environmental Molecular Sciences Laboratory, http://www.emsl.pnl.gov/

[37] Cybulski S M and Toczylowski R R 1999 J. Chem. Phys. 11110520

[38] Boys S F and Bernardi F 1970 Mol. Phys. 19553

[39] Alexander M H 1985 Chem. Phys. 92337

[40] Dubernet M-L, Flower D and Hutson J M 1991 J. Chem. Phys. 947602

[41] Delgado-Barrio G, Cortina A M, Varadé A, Mareca P, Villarreal P and Miret-Artés S 1986 J. Comput. Chem. 7208

[42] Secrest D and Eastes W 1972 J. Chem. Phys. 562502

[43] Muckerman J T 1990 Chem. Phys. Lett. 173200

[44] Smalley R F, Wharton L and Levy D H 1976 J. Chem. Phys. 643266

[45] Beswick J A and Delgado-Barrio G 1980 J. Chem. Phys. 73 3653

[46] Buchachenko A A, Prosmiti R, Cuhna C, Villarreal P and Delgado-Barrio G 2002 J. Chem. Phys. 1176117

[47] Kenny J E, Johnson K E, Sharfin W and Levy D H 1980 J. Chem. Phys. 721109

[48] Swartz B A, Brinza D E, Western C M and Janda K C 1984 J. Phys. Chem. 886272

[49] Nauta K and Miller R E 2002 J. Chem. Phys. 117 4846 